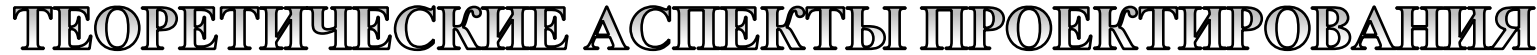

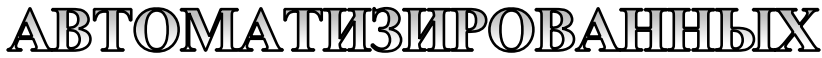

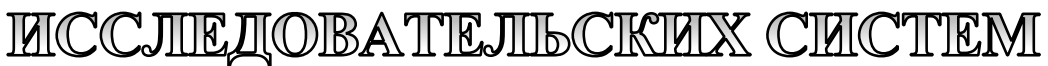

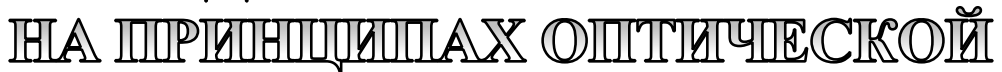

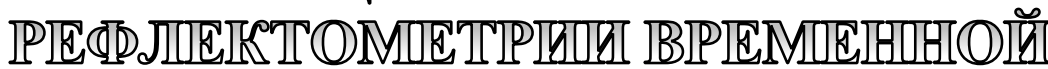

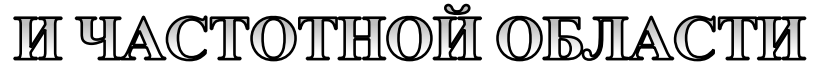

\author{
А.С. Смирнов, Пермский федеральныий исследовательский иеентр УрО РАН; Пермский \\ национальный исследовательский политехнический университет \\ А.И. Кривошеев, Пермский федеральный исследовательский центр УрО РАН; Пермский \\ национальный исследовательский политехнический университет, \\ Е.А. Носова, Пермский федеральныий исследовательский иентр УрО РАН; Пермский \\ национальный исследовательский политехнический университет \\ С.Д. Бочкова, Пермский федеральный исследовательский иентр УрО РАН; Пермский \\ начиональный исследовательский политехнический университет \\ Ф.Л. Барков, Пермский федеральный исследовательский иентр УрО РАН \\ В.В. Бурдин, Пермский федеральный исследовательский иентр УрО РАН; Пермский \\ национальный исследовательский политехнический университет \\ Ю.А. Константинов, Пермский федеральный исследовательский иентр УрО РАН
}

Рассмотрены теоретические аспекты проектирования автоматизированных исследовательских систем на принципах оптической рефлектометрии временной и частотной области. Представлены основные выражения для построения имитационных моделей оптических рефлектометров обоих видов. Изложен базовый принцип работы системы, представлены результаты функционирования ее отдельных модулей. Сделаны первичные выводы о работе элементов системы, определены ключевые направления деятельности.

Ключевые слова: оптическая рефлектометрия, метрология, автоматизация исследований, оптические волокна.

\section{Введение}

Серийное производство специальных волоконных световодов требует жесточайшего контроля эксплуатационных параметров последних на всех стадиях. Этому, в частности разработке автоматизированных систем анализа оптических параметров на ранних этапах технологического процесса (стадия изготовления заготовки - преформы оптического волокна), посвящен 8 ряд работ [1-3]. Немало статей посвящено и контролю оптико-геометрических параметров на завершающей стадии технологического процесса. Такой контроль уместно осуществлять при помощи методов оптической рефлектометрии $[4,6,7]$. Рефлектометрия временной области (OTDR - optical time domain reflectometry) широко применяется в производстве оптических волокон. Однако при этом данный 
метод имеет целый ряд существенных недостатков: мертвая зона «по событию», ограниченное длительностью импульса пространственное разрешение, нечувствительность к внешним воздействиям (что делает невозможными испытания в меняющихся условиях окружающей среды). Отчасти данные недостатки компенсирует применение рефлектометрии частотной области (OFDR - optical frequency domain reflectometry).

B отличие от техники OTDR данный способ исследования не использует импульсное зондирование - применяется частотное сканирование узкополосным непрерывным излучением. Выходными данными метода OFDR, в отличие от его предшественника (OTDR), является не временная развертка обратно-рассеянного импульса, а результат быстрого преобразования Фурье от функции непрерывной обратно-рассеянной мощности во времени, наглядно демонстрирующее своими спектральными пиками наличие отражений в исследуемом световоде.

Hе лишен недостатков и метод OFDR: например, достаточно высокая цена устройств, работающих по данному принципу. В некоторым смысле и положительной, и отрицательной чертой метода может быть поляризационная чувствительность. Отнесение данной особенности к позитивным или негативным факторам определяется в первую очередь задачами, стоящими перед производителями волокон или исследователями. Тем не менее многолетний опыт исследования оптико-геометрических параметров специальных волокон на разных стадиях свидетельствует в пользу необходимости создания метода одновременного использования двух техник в рамках одной автоматизированной системы. Теоретические основы проектирования подобной системы рассмотрены в данной работе.

\section{Моделирование}

В основу работы автоматизированной системы должно быть положено сравнение полученной рефлектограммы с эталонными данными. Их уместно получить при помощи имитационного моделирования. В случае с OTDR модель будет иметь следующие допущения [2]:

- волокно и световедущая жила внутри него обладают цилиндрической симметрией;

- волокно условно разделено на фрагменты, внутри которых оптические и геометрические свойства были приняты постоянными;

- отражения Френеля, идущие в направлении, противоположном направлению зондирования, не претерпевают вторичных отражений на пути следования;

- длина когерентности источника не превышает длины фрагмента;

- скорость звука в кварцевом стекле пренебрежимо мала по сравнению со скоростью света в этой же среде.

Известно, что пространственную (временну́ю) развертку мощности обратного рассеяния в момент времени $t_{n}$ в подобной системе можно представить как

$$
E_{n-1}=F_{0} b_{n-1} \Pi_{i=1}^{n}\left(f_{i}\right)^{2},
$$

где $E_{n-1}$ - уровень обратно-рассеянного излучения на фрагменте $(n-1) ; \mathrm{F}_{0}-$ уровень излучения, введенного в световод; $b$ и $f$ - коэффициенты, определяющие параметры отражения и затухания на заданном фрагменте. После логарифмирования полученную зависимость $\mathrm{E}_{n-1}(i)$ можно представить в виде рефлектограммы временной области.

Что касается области частотной, то математический аппарат OFDR в литературе описан по-разному $[4,6]$. Отчасти это связано с тем, что различные авторы концентрируют внимание на разных аспектах подобных моделей, интересующих их. Дадим в отношении данной модели те же допущения, что и для OTDR, сделав исключение лишь для длины когерентности источника - она должна быть, напротив, больше длины не только фрагмента, но и всей исследуемой линии. Итак, на основе упомянутых трактовок можно сформировать простой алгоритм, описывающий обратно-рассеянную мощность светового сигнала, пришедшего на фотоприемное устройство:

$$
E(\gamma t)=2 A K \cos \left(2 \gamma t\left|x-x_{r}\right|\right),
$$

где $y$ - частота излучения лазера в момент времени $t ; x_{\mathrm{r}}$ - координата нахождения 
зеркала в опорном плече OFDR; $x$ - текущая пространственная координата по длине волокна в момент времени $t$; $A$ - параметр, определяющий уровень сигнала в точке $x$ (зависит, в том числе, от показателей преломления зеркала в точке $x_{\mathrm{r}}$ и в волокне на координате $x$, а также от накопившихся к моменту $t$ потерь на рассеяние света). $K$ - коэффициент, характеризующий состояние поляризации света. Для системы, где происходит регистрация компонент для двух взаимно перпендикулярных состояний поляризации вводимого излучения, выражения примут вид

$$
\begin{aligned}
& E_{x}(\gamma t)=2 A_{x} K_{x} \cos \left(2 \gamma t\left|x-x_{r}\right|\right), \\
& E_{y}(\gamma t)=2 A_{y} K_{y} \cos \left(2 \gamma t\left|x-x_{r}\right|\right),
\end{aligned}
$$

где $E_{x}(y t)$ и $E_{y}(y t)$ - интерференционные сигналы, полученные на фотоприемных устройствах и описывающие изменение амплитуды электрического поля обратнорассеянного сигнала для поляризационных осей $x$ и $y$; $\mathrm{K}_{x}$ и $\mathrm{K}_{y}$ - величины, определяемые поляризационными неоднородностями как в исследуемом волокне, так и состоянием поляризации в опорном плече. Предположим, что данные неоднородности можно оценить при помощи матричного формализма Джонса [5]. Для этого представим анизотропный волоконный световод как последовательность повернутых относительно друг друга полупрозрачных фазовых пластинок, каждая из которых обладает индивидуальной анизотропией.

Матрица Джонса фазовой пластинки длиной $L$ с показателями преломления $n_{1}$ и $n_{2}$ (m-го участка волокна), как известно, в системе координат с осями, параллельными осям пластинки, имеет вид

$$
M_{m}=\left[\begin{array}{cc}
1 & 0 \\
0 & e^{-i \Delta \varphi}
\end{array}\right],
$$

где $\Delta \varphi=\frac{2 \pi L\left(n_{2}-n_{1}\right)}{\lambda}-$ фазовая задержка медленной компоненты волны, обусловленная разностью показателей преломления.

Поскольку рассматриваемый участок волокна ввиду закрутки повернут на определенный угол $\theta_{m}$ (пропорциональный расстоянию от конца волокна до участка, $\theta_{m}=\alpha L_{m}$, где $\alpha-$ коэффициент закрутки, в рад/км, $L_{m}$-пространственная координата $m$-го участка), в нормальной системе координат матрица Джонса преобразуется соответствующим образом:

$$
T_{m}=T_{\text {rot }}\left(\theta_{m}\right) M_{m} T_{r o t}\left(-\theta_{m}\right),
$$

где $T_{r o t}(\theta)=\left[\begin{array}{cc}\cos (\theta) & \sin (\theta) \\ -\sin (\theta) & \cos (\theta)\end{array}\right]-$ матрица поворота.

Следует также учесть, что при обратном распространении световой волны волокно становится закрученным в другую сторону, так что матрица Джонса того же участка волокна будет иметь несколько другой вид:

$$
T_{m}^{*}=T_{\text {rot }}\left(-\theta_{m}\right) M_{m} T_{r o t}\left(\theta_{m}\right) .
$$

Выражения для обратно-рассеянного линейно-поляризованного света (ввод излучения под 0 градусов) будет выглядеть следующим образом:

$$
\begin{aligned}
& {\left[\begin{array}{c}
E_{x(0)}(z) \\
E_{y(0)}(z)
\end{array}\right]=} \\
& =\left[\begin{array}{cc}
E x t_{p o l} & 0 \\
0 & 0
\end{array}\right] \Pi_{m=1}^{z} T_{m}^{*} \Pi_{m=z}^{1} T_{m}\left[\begin{array}{l}
1 \\
0
\end{array}\right],
\end{aligned}
$$

где $E x t_{p o l}$ - поляризационная экстинкция поляризатора LP. Полученные $E_{x(0)}(z)$ и $E_{y(0)}(z)$ уместно использовать для вычисления сигнала обратного рассеяния для случая частотной области. Тогда выражения (2) и (3) примут вид

$$
\begin{aligned}
& E_{x}(t)=2 A_{x} k E_{x(0)} \cos \left(2 \gamma t\left|x-x_{r}\right|\right), \\
& E_{y}(t)=2 A_{y} k E_{y(0)} \cos \left(2 \gamma t\left|x-x_{r}\right|\right),
\end{aligned}
$$

где $k$ - эмпирический коэффициент, определяемый уровнем введенного в исследуемое волокно сигнала. Очевидным является факт, что для производителей РМ-волокон и датчиков на их основе наиболее интересным с точки зрения регистрации $h$-параметра является рассмотрение выражения для $E_{y}(t)$, поскольку оно содержит компонент $E_{y(0)}$. Обычно в OFDR происходит вычисление векторной суммы, которая выглядит следующим образом:

$$
r(t)=\sqrt{\left|E_{x}(t)\right|^{2}+\left|E_{y}(t)\right|^{2}} .
$$

Для тестового моделирования использовался массив данных, обозначающих показатели преломления сердцевины оптического волокна и ряд других оптико- 
геометрических характеристик. В данном массиве были сознательно наведены три дефекта (локальные превышения показателя преломления) (рис. 1).

Далее было произведено моделирование двух видов рефлектометрии - временной и частотной. Результат можно увидеть на рис. 2 (графики незначительно смещены по оси ординат для наглядности).

Нижняя рефлектограмма (OFDR) получена после быстрого преобразования Фурье данных непрерывного обратного рассеяния; верхняя - методом простого логарифмирования данных обратного рассеяния, полученных при импульсном режиме работы.

\section{Принцип работы системы}

Упрощенный принцип функционирования системы изображен на рис. 3. Образец, поставляющийся в исследовательскую лабораторию, коммутируется с оптическим входом системы одним из торцов. Обычно используется внешний (свободный) торец оптического волокна его достаточно легко стыковать или сваривать с другими световодами. Его спецификация, содержащая результаты исследования оптико-геометрических параметров на более ранних стадиях, загружа- ется в систему. На базе полученных данных осуществляется моделирование с использованием математического аппарата, описанного выше. Рефлектограммы, полученные методами OFDR и OTDR, загружаются в виде дискретных массивов в модуль корреляции, где они проходят совместную обработку с данными моделирования.

Далее возможно различное развитие событий: либо вычисление коэффициента взаимной корреляции осуществляется в сканирующем окне с определением дефекта пороговым алгоритмом и автоматизированным принятием решения, либо производится визуализация трехмерной коррелограммы (рис. 4). По оси абсцисс отложена пространственная координата (м), по оси ординат - размер сканирующего окна (м), цветовой информацией в градациях серого отображена величина коэффициента корреляции (белый цвет - коэффициент взаимной корреляции равен 1 , черный -0 ).

Данная опция предполагается в режимах обучения системы, когда на визуализированной коррелограмме исследователь во время первых измерений сам находит проблемные участки. В дальнейшем система должна автоматически, без участия человека, находить подобные дефекты

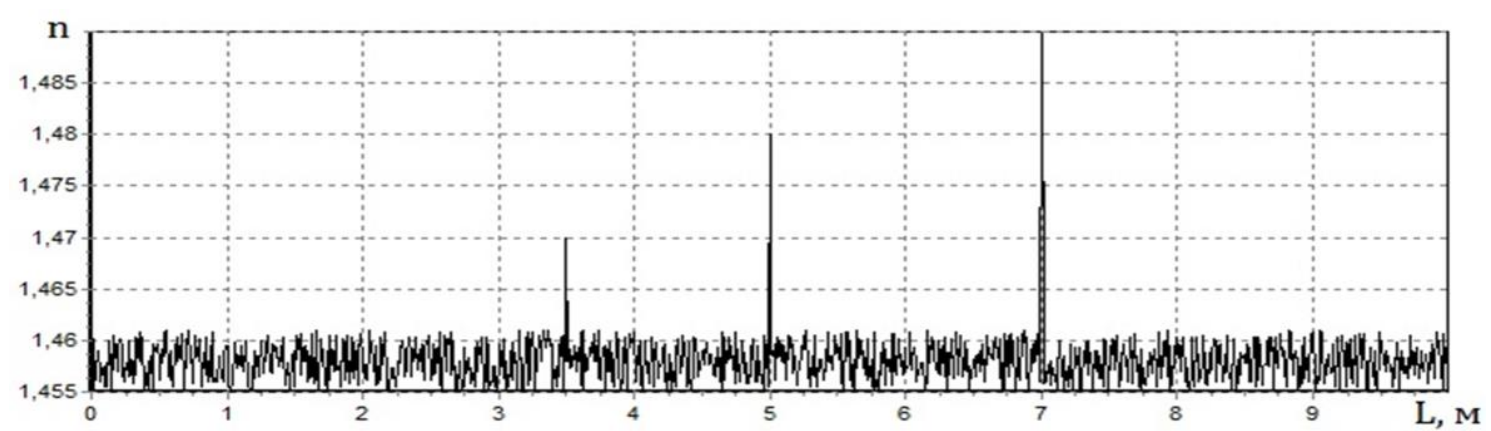

Рис. 1. Массив данных, характеризующих среду рассеяния света с тремя артефактами

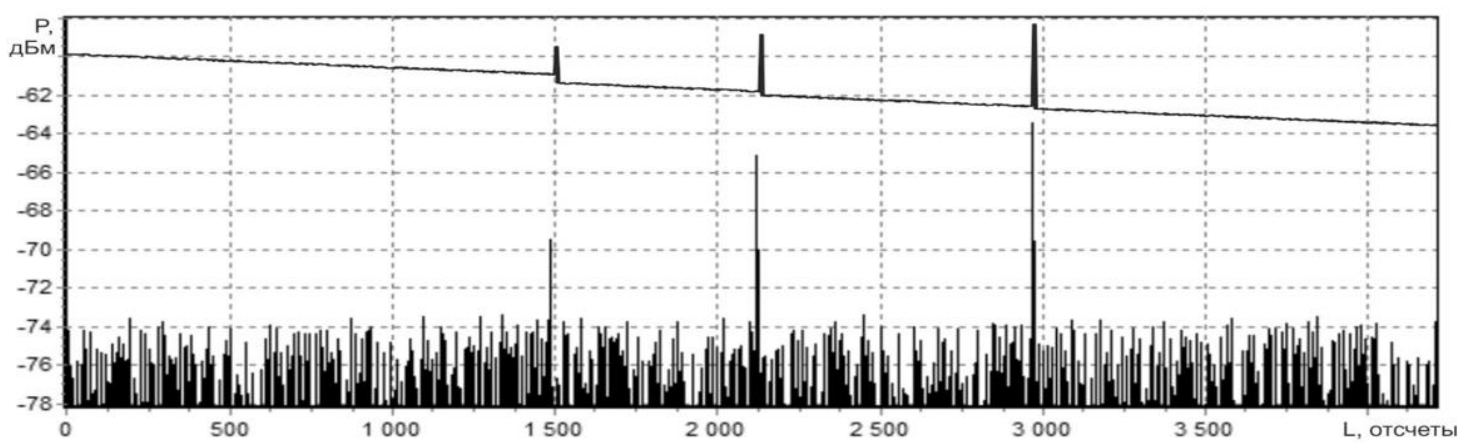

Puc. 2. Две смоделированные рефлектограммы (верхняя-OTDR, нижняя-OFDR) 


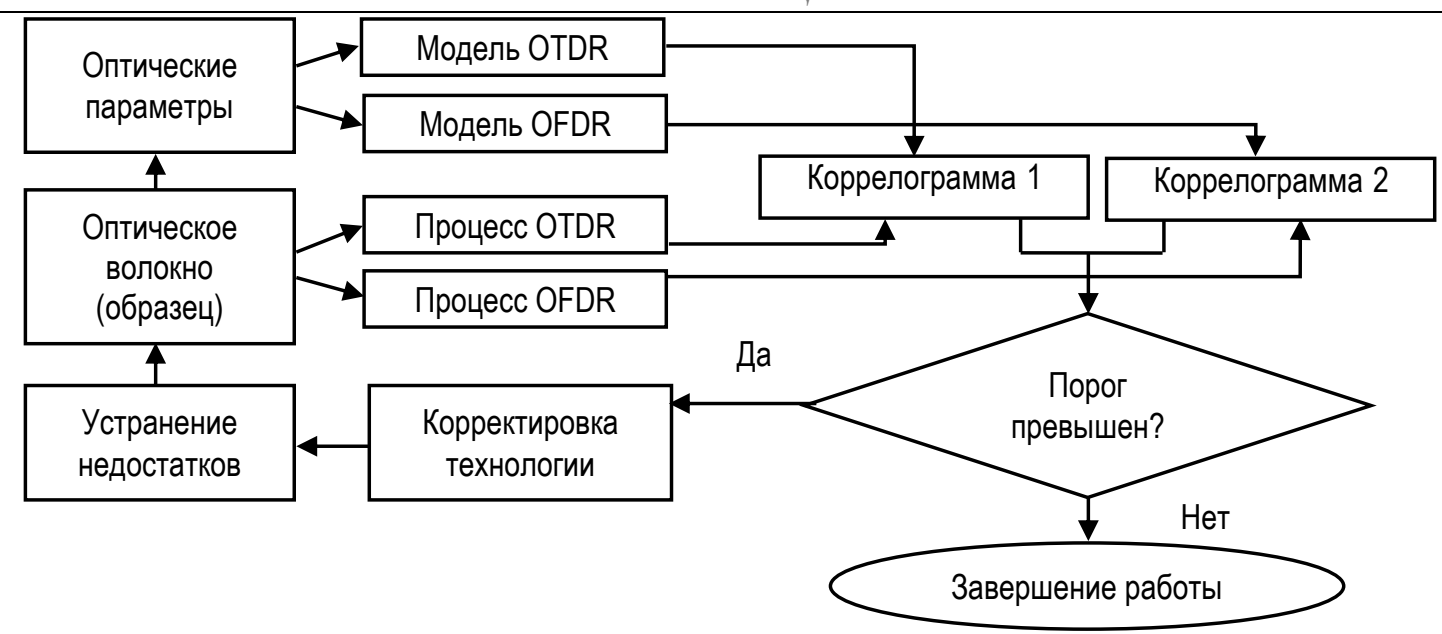

Рис. 3. Упрощенный принцип функционирования системы

в волокнах того или иного типа (на которых произведено обучение системы).

\section{Выводы и будущие работы}

После проведения серии компьютерных экспериментов стало очевидно, что данные сразу с двух устройств (OFDR и OTDR) не только дают более полную картину, пригодную для автоматизированного принятия решения о кондиционности того или иного образца оптического волокна, но и пригодны для комплексирования с целью получения информации иного типа. Например, скорость частотной перестройки в устройстве OFDR составляет обычно десятки герц (Гц). Это становится неприемлемым, когда ставится задача вибрационных испы-

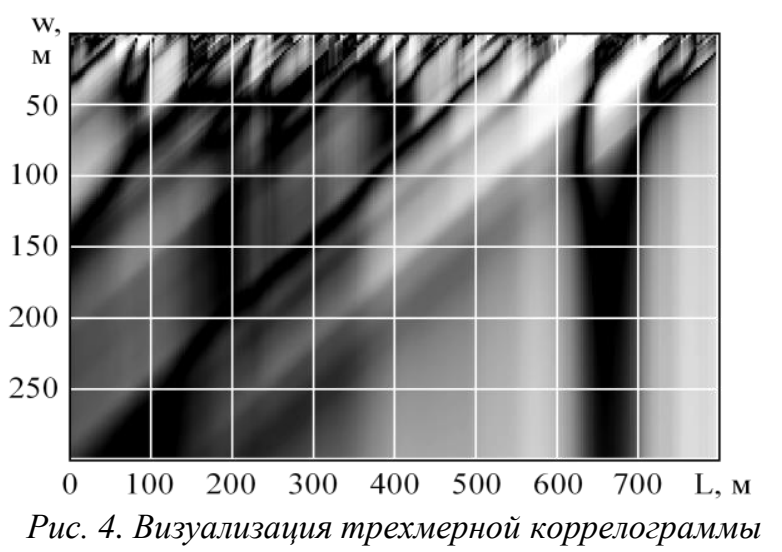

таний устройства, содержащего оптические волокна. Способ OTDR может обеспечивать скорость работы, позволяющую получать данные с частотой в десятки килогерц (кГц) - на три порядка больше. Другая задача - комплексирование данных с двух рефлектограмм. На рефлектограммах частотной области достаточно хорошо детализированы мелкие неоднородности, рефлектограммы же области временной - напротив, более сглаженные, с хорошо заметным рельефом длиннопериодных колебаний. Таким образом, получая две рефлектограммы разных типов, представляются возможными и необходимыми частотная фильтрация и дальнейшая суперпозиция последних, что может стать направлением дальнейшей деятельности. Еще одной задачей будущей работы станет объединение разработанных модулей в действующую на серийном производстве программноаппаратную систему и оценка ее эффективности.

Авторы благодарят Публичное акционерное общество «Пермская научно-производственная приборостроительная компания» (ПАО «ПНППК») за предоставленные образцы, помощь в организации экспериментов, а также инженера лаборатории фотоники Пермского федерального исследовательского центра УрО РАН (ПФИЦ УрО РАН) Д. Клода за плодотворные дискуссии.

\section{Библиографический список}

1. Автоматизированный сбор данных при исследовании характеристик волоконных световодов на этапах производства / Ю.А. Константинов, И.И. Крюков, М.М. Поскребышев, Н.А. Харламова // Научно-технические ведомости СПбГПУ. Информатика. Телекоммуникации. Управление. - 2011. № 6-2(138). - С. 30-34.

2. Константинов Ю.А., Первадчук В.П. Автоматизация производства специальных волоконных световодов с применением эмуляторов физических процессов обратного рассеяния // Автоматизация и современные технологии. - 2013. - № 8. - С. 3-9. 
3. Телевизионная система измерения размеров заготовки волоконных световодов в ходе процесса химического парофазного осаждения / Ю.А. Константинов, И.И. Крюков, М.М. Поскребылеев, H.A. Харламова // Научно-технические ведомости СПбГПУ. Информатика. Телекоммуникации. Управление. - 2010. - № 6(113). - С. 155-158.

4. Jasenek J., Cermak $O$. Optical reflectometry with synthesized coherence function, Proceedings Volume 4016, Photonics, Devices and Systems; (1999) / Photonics Prague '99, 1999, Prague, Czech Republic. URL: https://doi.org/10.1117/12.373624.

5. Jones R.C. New calcules for the treatment of optical systems. Г-VIII, J. Opt. Soc. Amer. - 1941. - Vol. 31. P. 488; 1948. - Vol. 38. - P. 671; 1956. - Vol. 46. - P. 126.

6. Soller B., Gifford D., Wolfe M., Froggatt M. High resolution optical frequency domain reflectometry for characterization of components and assemblie // Opt. Express. - 2005. - 13(2) - P. 666-674.

7. Soller B.J., Wolfe M., Froggatt M.E. Polarization resolved measurement of Rayleigh backscatter in fiberoptic components, January 2005. URL: https://www.researchgate.net/publication/229014677_ Polarization_resolved_measurement_of_Rayleigh_backscatter_in_fiber-optic_components.

\title{
THEORETICAL ASPECTS OF THE DESIGN OF AUTOMATED RESEARCH SYSTEMS BASED ON THE PRINCIPLES OF OPTICAL REFLECTOMETRY IN THE TIME AND FREQUENCY DOMAINS
}

\author{
A.S. Smirnov ${ }^{1,2}$, A.I. Krivosheev ${ }^{1,2}$, E.A. Nosova ${ }^{1,2}$, S.D. Bochkova ${ }^{1,2}$, \\ F.L. Barkov ${ }^{1,2}$, V.V. Burdin ${ }^{1,2}$, Yu.A. Konstantinov ${ }^{1}$ \\ ${ }^{1}$ Perm Federal Research Center UB RAS \\ ${ }^{2}$ Perm National Research Polytechnic University
}

The paper discusses the theoretical aspects of the design of automated research systems based on the principles of optical reflectometry in the time and frequency domains. The basic expressions for the construction of simulation models of optical reflectometers of both types are presented. The basic principle of the system is described, the results of the functioning of its separated modules are presented. Some initial conclusions on the work of the elements of the system were made, key areas of activity were identified.

Keywords: optical reflectometry, metrology, research automation, optical fibers.

\section{Сведения об авторах}

Смирнов Александр Сергеевич, младший научный сотрудник лаборатории фотоники, Пермский федеральный исследовательский центр УрО РАН (ПФИЦ УрО РАН), 614900 , г. Пермь, ул. Ленина, 13А; заведующий учебной лабораторией кафедры «Прикладная математика», Пермский национальный исследовательский политехнический университет (ПНИПУ), 614990, г. Пермь, Комсомольский пр., 29; e-mail: a.s.smrnv@ gmail.com

Кривошеев Антон Иванович, младший научный сотрудник лаборатории фотоники, ПФИЦ УрО РАН; аспирант, ПНИПУ; e-mail: antokri @ yandex.ru

Носова Екатерина Александровна, младший научный сотрудник лаборатории фотоники, ПФИЦ УрО РАН; аспирант, ПНИПУ; e-mail:space.kattie@gmail.com

Бочкова Софья Дмитриевна, младший научный сотрудник лаборатории фотоники, ПФИЦ УрО РАН; аспирант, ПНИПУ; e-mail:sooyfar@gmail.com

Барков Федор Леонидович, кандидат физико-математических наук, старший научный сотрудник лаборатории фотоники, ПФИЦ УрО РАН; доцент кафедры «Общая физика», ПНИПУ; e-mail: fbarkov@pstu.ru

Бурдин Владислав Викторович, кандидат физико-математических наук, старший научный сотрудник лаборатории фотоники, ПФИЦ УрО РАН; доцент кафедры «Общая физика», ПНИПУ; e-mail: vlaburdi@mail.ru

Константинов Юрий Александрович, кандидат технических наук, научный сотрудник лаборатории фотоники, ПФИЦ УрО РАН; e-mail: yuri.al.konstantinov@ro.ru 